\title{
Prediction-based feedback control of a class of processes with input-varying delay
}

\author{
Delphine Bresch-Pietri, Jonathan Chauvin, Nicolas Petit
}

\begin{abstract}
At the light of a simple tutorial example, this paper discusses the merits of a recently introduced technique to control a class of systems with a delay depending on the past values of the control variables. The relations of the proposed technique with previous works from the literature on predictorbased controllers are discussed. The treated example is representative of a wide class of systems often observed in process control and distributed parameter systems. The anticipating capabilities of the proposed controller yields interesting closedloop performance.
\end{abstract}

\section{INTRODUCTION}

With the still increasing expectations of dynamic performances, a consequent number of industrial applications does require output feedback control laws, often involving a physical dead time, as the available actuators can be located far away from the main considered plant. This results into a temporal lag, which can occur in various contexts such as in automotive engine [2], mixing processes for chemical reactors [9], blending [6] or batch processes [21] or even heater collector plant [23]. As is reported in Table I, these industrial processes often present transportation of material, which can be directly depending on the manipulated variable. Therefore, the introduced delay is inherently an inputdependent time delay, which increases the complexity of the control task.

One of the most simple example of this type of nonlinear dynamical systems one can think of is the temperature regulation of the shower system, or bath depicted in Fig. 1 : the involved non-negligible pipe holdups are directly correlated to the history of the input flow rates. Due to its relative simplicity and its occurrence in everyday life, it is often used to introduce time-delay systems in lectures [28] and to illustrate some corresponding control challenges. Surprisingly, as similar problems involving input-varying delay, such as the crushing-mill presented in [22], this problem has never been studied theoretically at our knowledge. Of course, relatively simple practical solutions such as thermostatic mixers and shower valve controllers exist. Yet, the coupling with the output dynamics is very troublesome. This implies the consideration of the stabilization of a non-linear process, with input-dependent time-delay in the input.

D. Bresch-Pietri (corresponding author) is a $\mathrm{PhD}$ Candidate in Mathematics and Control at MINES ParisTech, 60, Bd St-Michel, 75272 Paris, Cedex 06, France. Email : delphine.bresch-pietri@mines-paristech.fr

J. Chauvin is with the Département Contrôle, Signal et Système in IFP Energies Nouvelles, 1 et 4 Avenue du Bois Préau, 92852 Rueil Malmaison, France

N. Petit is with the Centre Automatique et Systèmes, Unité Mathématiques et Systèmes at MINES ParisTech, 60 Bd St Michel, 75272 Paris, France
A classically considered approach in such situation (see the discussion in [26]) is to treat the delay dependence on the control by a robustness approach, i.e. by neglecting this dependance.

The robust stability of systems with time-varying delay in the input have been widely studied lately : using either a Lyapunov-Razumikhin function or a Lyapunov-Krasovskii functional, delay-dependent stability criteria are obtained under the form of Linear Matrix Inequalities (LMIs) [8] [10] [12] [17]. The "memoryless" controllers employed in such approaches are relatively easy to implement. Yet, to improve closed-loop dynamic performance, one would prefer to use a predictor-based control law [1] [18] [25] aiming at compensating the delay via a distributed delay of infinite dimension. Such techniques, which are widely used for a constant input time-delay (see for instance [11] [14] [19] [20] or [7] [22] and the reference therein) are less popular for time-varying ones. Recently, some have been developed in such a framework in [27], where substantial LMIs have to be checked, and in [15], where the invertibility of a certain delay-operator is assumed. To the best of the authors knowledge, the relation between the above material and the stabilization of input-varying delay systems has not been studied.

In this paper, we follow the overture proposed in [13] and [15] to analyze the stability of linear input time-delay systems. These newly proposed techniques have been developed in [3] [4] [5] [16] to address uncertainties to constant input time-delays in various contexts. Here, we extend these tools to linear systems with time-varying delay in the input, similarly to what was done in [15], and explicitly relate the obtained result with the regulation of a class of inputvarying delay processes to address the particular problem under consideration in a tutorial spirit. These are the main contributions of the paper.

The paper is organized as follows. In Section II, we describe the mixing process under consideration, before designing in Section III a prediction-based controller using a tailored change of time scale. After having presented and commented in Section IV some numerical results, the proof of convergence of the proposed controller is provided in Section V.

\section{Problem statement}

Consider the bath system represented in Fig. 1, where the average bath temperature is the result of a mixing between a cold water source (flow rate $u_{1}$ and temperature $T_{1}$ ) and a 


\begin{tabular}{llll}
\hline Process & State variable & Control variable & Transport delay source \\
\hline $\begin{array}{l}\text { Air-Fuel Ratio control } \\
\text { in SI Engines }\end{array}$ & Exhaust Air-Fuel Ratio & Injected mass of fuel & $\begin{array}{l}\text { Pipe transportation from } \\
\text { the injector to the exhaust } \\
\text { Lambda Sensor }\end{array}$ \\
\hline $\begin{array}{l}\text { Exhaust Gas Recirculation } \\
\text { in SI Engines }\end{array}$ & Intake Burned Gas Rate & $\begin{array}{l}\text { Recirculated burned gas } \\
\text { flow rate }\end{array}$ & $\begin{array}{l}\text { Pipe transportation } \\
\text { into the intake line }\end{array}$ \\
\hline $\begin{array}{l}\text { Blending systems } \\
\text { in refineries }\end{array}$ & $\begin{array}{l}\text { Final production tank } \\
\text { properties }\end{array}$ & Components flow rates & $\begin{array}{l}\text { Pre-blend volumes } \\
\text { and pipe transportation }\end{array}$ \\
\hline Solar collector fields & Output temperature & Input flow rate & $\begin{array}{l}\text { Pipe transportation } \\
\text { in the outlet tube }\end{array}$ \\
\hline Bath/Shower & Output temperature & Input flow rates & Pipe transportation \\
\hline
\end{tabular}

TABLE I

COMPARISON OF SOME PROCESSES WITH TRANSPORT DELAY.

warm one $\left(u_{2}, T_{2}\right)$. For comfort purposes, the user of the bath wishes to obtain a desired temperature $T_{r e f}$, without over- or under-shoots, and as fast as possible (namely, close to the minimum reaching time introduced by the transport delay through the pipe).

For sake of simplicity, we assume here that the change of the faucet position is immediate and that the cold source flow rate is constant. Assuming that the position of the warm water faucet is directly correlated to the flow rate through static relations, $u_{2}$ could be then considered as the input variable. Finally, we assume that the bath temperature $T_{f}$ is available for measurement and that the bath volume is constant (i.e. $\forall t \geq 0, u_{\text {out }}(t)=u_{1}+u_{2}(t)$ ).

\section{A. Balance equations}

Assuming that the mixing at the node is instantaneous, the punctual temperature at this node can simply be expressed as

$$
T_{m o y}(t)=\frac{u_{1} T_{1}+u_{2}(t) T_{2}}{u_{1}+u_{2}(t)}
$$

We neglect the heat transfer during the flow transport from the node to the pipe output, namely

$$
T_{\text {out }}(t)=T_{\text {moy }}(t-D(t))
$$

where $D(t)$ accounts for the varying transport delay implicitly defined by

$$
V_{P}=\int_{t-D(t)}^{t}\left(u_{1}+u_{2}(s)\right) d s
$$

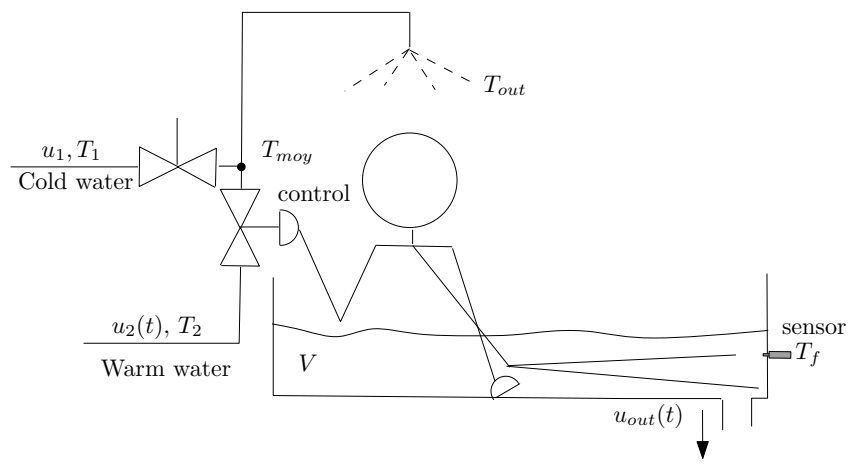

Fig. 1. The studied shower system. with $V_{P}$ the pipe volume. Further, considering the bath volume $V$ as constant, a heat balance yields, using (2),

$$
\frac{d}{d t}\left(T_{f}\right)=\frac{u_{1}+u_{2}(t)}{V}\left[-T_{f}(t)+T_{m o y}(t-D(t))\right]
$$

\section{B. Constraints and control objective}

a) Output temperature: To avoid to be scaled, the output water temperature needs to be upper-bounded. Simultaneously, for comfort purposes, it also has to be lowerbounded : $\underline{T} \leq T_{\text {out }} \leq \bar{T}$.

b) Valve Position: Both faucets admit maximal and minimal positions and, therefore, the warm water flow rate is also bounded, i.e. $u_{2} \in\left[0, \overline{u_{2}}\right]$.

c) Control objective: The control objective is to have system (4) to track the given temperature set-point $T_{\text {ref }}$ as fast as possible, taking into account the above constraints. To reach this goal, we aim at developing a prediction-based control law taking advantage of the knowledge of the implicit delay variation law (3).

\section{Control Design}

For sake of clarity, without loss of generality, in this section, we normalize the quantity introduced above as $T_{1}=0, T_{2}=1, u_{1}=1, V=1, T_{r e f} \in[0,1[$ and denote now $u=u_{2} \in[0, \bar{u}]$ the actual actuator.

\section{A. Alternative system representation}

In order to design an explicit prediction-based feedback law, we first introduce the following change of time

$$
\tau \stackrel{\text { def }}{=} h(t)=\frac{1}{1+\bar{u}} \int_{0}^{t}(1+u(s)) d s+\tau_{0}
$$

where $\bar{u}$ is a normalization factor chosen as $\bar{u}=\frac{T_{\text {ref }}}{1-T_{\text {ref }}}$ and where $\tau_{0} \geq 0$ is a given constant. This leads to the alternative linear system

$$
\frac{d X}{d \tau}(\tau)=(1+\bar{u})\left[-X(\tau)+T_{m o y}\left(\tau-D_{2}(\tau)\right)\right]
$$

where the alternative system state is defined as $X(\tau)=T_{f}(t)$ and a new delay has been introduced as

$$
D_{2}(\tau)=\tau-t+D(t)=\tau-h^{-1}(\tau)+D\left(h^{-1}(\tau)\right)
$$


In this new time scale ${ }^{1}$, (6) is a linear input-delay system with constant parameters. Rewriting the alternative delay as $D_{2}(h(t))=h(t)-t+D(t)$ and considering both (3) and (5), one can observe that this delay is still input-dependent, but in a much more complex way. Before working with this representation, we need to make sure (5)-(7) is well-posed.

(i) The function $h$ defined in (5) is a $C^{1}$ function, strictly increasing w.r.t. $t$. Therefore, it describes a diffeomorphism and, in particular, its inverse in (7) is well-defined.

(ii) One has to ensure that the delay $D_{2}$ is well-defined, namely positive, i.e. that $\forall t \geq 0, \tau \geq t-D(t)$. This property is given by choosing $\tau_{0}$ large enough compared to the delay upper bound $\bar{D}=V_{P}$.

(iii) $h$ is unbounded and then $\lim _{t \rightarrow \infty} T_{f}(t)=\lim _{\tau \rightarrow \infty} X(\tau)$ (if it exists). This enables a direct translation of any asymptotic result obtained for the alternative plant (6) on the original one (4).

\section{B. Control design for the alternative system}

We now formulate the following theorem, a proof of which is provided in Section V.

Theorem 1: Consider the closed-loop system

$$
\begin{aligned}
& \dot{X}(t)=A X(t)+B U(t-D(t)) \\
& U(t)=U^{r}-K X^{r}+K\left[e^{A D(t)} X(t)+\int_{t-D(t)}^{t} e^{A(t-s)} B U(s) d s\right]
\end{aligned}
$$

where $X \in \mathbb{R}^{n}, U$ is scalar, the pair $(A, B)$ is controllable, $K$ is chosen such that $A+B K$ is Hurwitz, $\left(X^{r}, U^{r}\right)$ is a given equilibrium set-point and $D: \mathbb{R}_{+} \rightarrow[\underline{D}, \bar{D}]$ is a timedifferentiable function. Then, there exists $\left.\delta^{*} \in\right] 0 ; 1[$ such that, provided

$$
\forall t \geq 0, \quad|\dot{D}(t)|<\delta^{*},
$$

the considered plant exponentially converges to the equilibrium $X^{r}$.

Before studying the application of this theorem to our mixing process, a few comments can be made about it.

Control law (8b) is directly inspired by the constant delay case, calculating the state prediction over a time window of varying length $D(t)$. Of course, exact compensation of the delay is not achieved with this controller. To do so, one would need to consider a time window of which length would match exactly the value of the future delay, as it is made in $[15]^{2}$. In other words, this requires to be able to predict the future variation of the delay, which is not always practically achievable for an input-varying delay.

In this context, (9) can be interpreted as a condition for robust compensation achievement. Namely, if the delay

\footnotetext{
${ }^{1} \mathrm{~A}$ different constant scale factor could be introduced in (5) to simplify the expression of (6) at the expense of later complexity in the analysis of closed-loop behavior.

${ }^{2}$ In details, defining the delay operator $\phi(t)=t-D(t)$ and assuming that its inverse exists and is available, exact delay-compensation is obtained with the feedback law $U(t)=K X\left(\phi^{-1}(t)\right)$ where the prediction can be written as $X\left(\phi^{-1}(t)\right)=\left[e^{A\left(\phi^{-1}(t)-t\right)}+\int_{t}^{\phi^{-1}(t)} e^{A\left(\phi^{-1}(t)-s\right)} B U(\phi(s)) d s\right]$.
}

varies sufficiently slowly, its current value $D(t)$ used for prediction will be close enough to its future values, and the corresponding prediction will be accurate enough to ensure the stabilization of the plant.

Interestingly, the exact same condition is stated in [27], where the delay is also assumed to be time-differentiable. Yet, the approach designed in [27] differs from ours in the way that a constant average delay value is used for control ${ }^{3}$, which should naturally result into poorer performance than the proposed one.

\section{Control law for the original plant}

Furthering Theorem 1, we now use the following controller.

$$
\begin{aligned}
u(t) & =\operatorname{Sat}_{[0, \bar{u}]}\left\{\frac{T_{\text {moy }}(t)}{1-T_{\text {moy }}(t)},\right\} \\
T_{\text {moy }}(t) & =(1+k) T_{\text {ref }}-k\left[e^{-(1+\bar{u}) D_{2}(t)} T_{f}\left(h^{-1}(t)\right)\right. \\
+ & \left.(1+\bar{u}) \int_{t-D_{2}(t)}^{t} e^{-(1+\bar{u})(t-s)} T_{\text {moy }}(s) d s\right] \\
D_{2}(t) & =t-h^{-1}(t)+D\left(h^{-1}(t)\right)
\end{aligned}
$$

where the function $h$ has been defined in (5) and $\operatorname{Sat}_{[0, \bar{u}]}$ represents the saturation operator.

Indeed, the alternative plant (6) directly fulfills the framework of Theorem 1. As a result, to obtain convergence with the prediction-based control law (11), which is a direct application of (8b) to the considered case, a sufficient condition is

$$
\left|\frac{\partial D_{2}}{\partial \tau}(\tau)\right|<\delta^{*}
$$

First, from (7) and (5), one can easily obtain an analytic expression of this partial derivative

$$
\frac{\partial D_{2}}{\partial \tau}(\tau)=\frac{\partial D_{2}}{\partial \tau}(h(t))=1-[1-\dot{D}(t)] \frac{1+\bar{u}}{1+u(t)}
$$

Further, taking a time-derivative of the implicit equation (3), one gets, with normalized data,

$$
1-\dot{D}(t)=\frac{1+u(t)}{1+u(t-D(t))}
$$

and then, substituting,

$$
\begin{aligned}
\left|\frac{\partial D_{2}}{\partial \tau}(\tau)\right| & <\delta^{*} \Leftrightarrow\left|1-\frac{1+\bar{u}}{1+u(t-D(t))}\right|<\delta^{*} \\
& \Leftrightarrow \frac{1+\bar{u}}{1+\delta^{*}}<1+u(t-D(t))<\frac{1+\bar{u}}{1-\delta^{*}} \\
& \Leftrightarrow \frac{1-\delta^{*}}{1+\bar{u}}<1-T_{\text {moy }}(t-D(t))<\frac{1+\delta^{*}}{1+\bar{u}}
\end{aligned}
$$

\footnotetext{
${ }^{3}$ More precisely, the control law is designed after a reduction model using this average value.
} 


\begin{tabular}{|c|c|c|}
\hline & Notation & Value \\
\hline Cold water temperature & $T_{1}$ & $20{ }^{\circ} \mathrm{C}$ \\
\hline Warm water temperature & $T_{2}$ & $40{ }^{\circ} \mathrm{C}$ \\
\hline Maximum flow rate & $\overline{u_{2}}$ & $0.25 \mathrm{~L} / \mathrm{s}$ \\
\hline Cold water flow rate & $u_{1}$ & $0.125 \mathrm{~L} / \mathrm{s}$ \\
\hline Pipe volume & $V_{P}$ & $6.3 \mathrm{~L}$ \\
\hline Bath volume & $V$ & $100 \mathrm{~L}$ \\
\hline Maximum admissible temperature & $\bar{T}$ & $40{ }^{\circ} \mathrm{C}$ \\
\hline Minimum admissible temperature & $\underline{T}$ & $15{ }^{\circ} \mathrm{C}$ \\
\hline
\end{tabular}

TABLE II

BATH SYSTEM PARAMETERS USED FOR SIMULATION.

Finally, exploiting the equilibrium relation between $\bar{u}$ and $T_{r e f}$, this condition can be rewritten as the inequality bearing on the input

$$
\left|T_{\text {moy }}(t-D(t))-T_{\text {ref }}\right|<\delta^{*} \frac{1}{1+\bar{u}}
$$

Expanding (11), one can recast this inequality into a simple inequality bearing on the gain $k$. Indeed,

$$
\left|T_{\text {moy }}(t)-T_{\text {ref }}\right| \leq k\left[2+(1+\bar{u})\left(\tau_{0}+\bar{D}\right)\right] \bar{T}
$$

Consequently, by choosing

$$
k<k_{\text {max }}=\frac{\delta^{*}}{\bar{T}(1+\bar{u})\left(2+(1+\bar{u})\left(\tau_{0}+\bar{D}\right)\right)}
$$

condition (13) is satisfied. Finally, as the diffeomorphism $h$ is unbounded, the asymptotic convergence of the system state in (6) to $T_{r e f}$ involves the one of $T_{f}$.

This result is not surprising : Theorem 1 requires the delay to vary sufficiently slowly and the delay variations implicitly depend on the control input. Then, restricting the input variations by choosing the feedback gain sufficiently small seems like a natural solution. Further, as the mixing process under consideration is stable, we can arbitrarily choose $k$ as small as desired and in particular to satisfy (13).

An important point for implementability is the causality of the control law (10), as the inverse transformation $h^{-1}$ is employed. In other words, the following property has to be satisfied : $\forall t \geq 0, \quad h^{-1}(t) \in[0, t]$. Considering (5), one can easily see that, for $T_{r e f} \geq T_{f}(0)$, this property is satisfied for all $\tau_{0} \geq 0$ as the integrand is greater than one. On the other hand, for $T_{r e f}<T_{f}(0)$, one has simply to choose $\tau_{0}$ large enough to ensure this.

For strict implementability, an expression of the bound $\delta^{*}$ is provided in Section V. Nevertheless, as this formulation is obtained based on a Lyapunov analysis, it turns out to be quite conservative, as the above simulation results emphasize it and does not aim at being used in practice.

\section{Simulation RESUlts}

In this section, we provide some numerical results to illustrate the merits of the proposed approach. As well as our prediction-based controller, we consider a "memoryless" control using a simple proportional feedback. Both are compared to open-loop performances.
The parameters of the bath system used for simulation are provided in Table IV. We aim at controlling the system from an equilibrium point where the bath is filled only by the cold water source, namely $T_{f}(0)=20^{\circ} \mathrm{C}$, up to $T_{\text {ref }}=30^{\circ} \mathrm{C}$. Figure 2 shows a comparison between the three aforementioned strategies, with the same feedback gain $k=10$. One can see that both feedback strategies provide, predictably, a significant performances improvement. In particular, the proposed controller favorably compares to a simple proportional one in terms of output variations and overall effect. In details, both increase the warm water flow rate $u_{2}$, aiming at providing a warm input into the bath, which results into a delay decrease (around 10s less than with an open-loop strategy).

In the light of this result, as the two feedback laws act quite similarly, one may prefer to use the proportional feedback law, much easier to implement. Yet, the merits of the proposed prediction-based law are highlighted while increasing the feedback gain $k$. Indeed, quickly, damped oscillations appear using a proportional control, the damping decreasing as the gain increases. Finally, for a gain value $k=26$, a limit cycle is reached and stabilization cannot be achieved as can be observed in Fig. 3. This can be easily interpreted analyzing the characteristic equation of the closed-loop alternative system (6)

$$
\Delta(\lambda)=\lambda+\frac{u_{1}+\overline{u_{2}}}{V}\left(1+k e^{-\lambda D_{2}}\right)=0
$$

As it is well-known [24], the (infinite) characteristic roots are all located in the right-hand complex half-plan if and only if the following condition is satisfied

$$
-1<k<\frac{V}{\left(u_{1}+\overline{u_{2}}\right) D_{2}} \sqrt{z_{1}^{2}+\left(\frac{u_{1}+\overline{u_{2}}}{V}\right)^{2} D_{2}{ }^{2}}
$$

where $z_{1}$ is the unique solution of $\tan (z)=-\frac{V}{\left(u_{1}+\overline{u_{2}}\right) D_{2}} z$ on the interval $(\pi / 2, \pi)$. This range of variation is represented in Fig. 4, for a delay varying between $15 s$ and $35 s$ (corresponding to the range of the delay oscillations in Fig. 3). The value of the maximum stabilizing gain for a $25 \mathrm{~s}$ delay (the delay steady-state value of the considered operating point) is circled in red. While increasing the proportional gain $k$ from 25 to 26 , one can observe that the upper unstable region is reached, generating the behavior in Fig. (3). On the other hand, the prediction-based control still achieves honorable performances for such a feedback gain, as it is well-tailored. If the actuator was not saturated, one would reasonably expect transient dynamics improvements.

Finally, calculating the expression (19) of $\delta^{*}$ provided below, one obtains a scale of $10^{-6}$ which would result here into a gain limitation around $10^{-7}$ as the initial error tracking of the bath temperature is 10 degrees. This value is of course extremely conservative as the above simulation results underline it. Nevertheless, this expression leads to the conclusion, at least according to the Lyapunov proof, that the faster the dynamics of the system is, the smaller this bound 


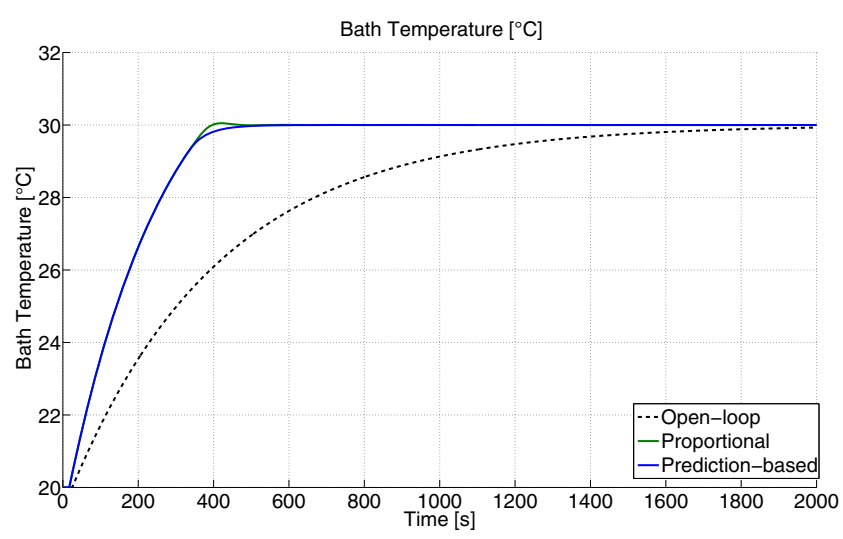

Warm water Flow rate $[\mathrm{L} / \mathrm{s}]$
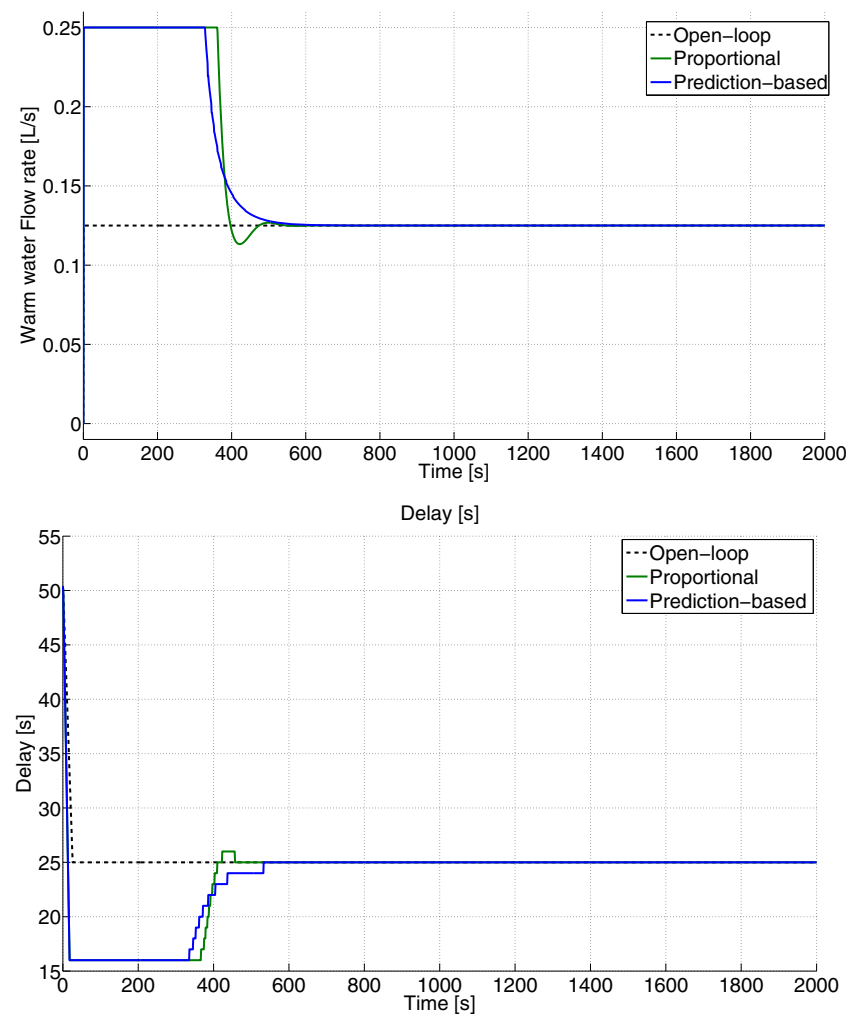

Fig. 2. Stabilization of the bath temperature at the equilibrium $T_{\text {ref }}=30^{\circ} \mathrm{C}$, starting from $T_{f}(0)=20^{\circ} \mathrm{C}$ respectively without feedback (black dotted) and with a gain $k=10$ both for proportional (in green) and predictionbased (in blue) feedback.

will be ${ }^{4}$. We now detail this proof.

\section{Proof of THEOREM 1}

In the following, we use the Lyapunov tools introduced in [13] to analyze the stability of input time-delay systems and which are based on a backstepping transformation of a

${ }^{4}$ In details, the constant $M_{1}, M_{2}$ and $M_{3}$ introduced below are then larger, which results into a smaller value of $\delta^{*}$.
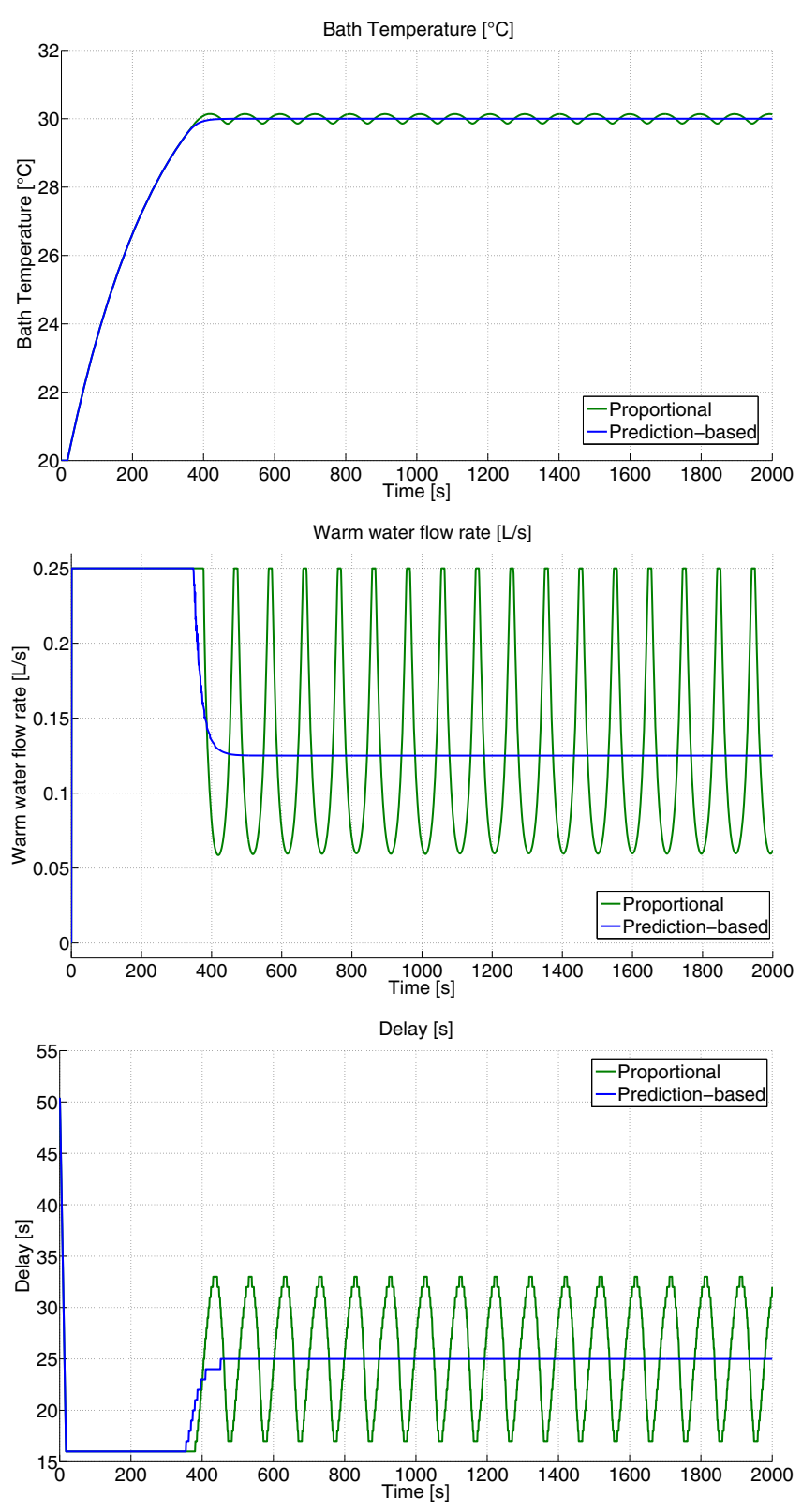

Fig. 3. Stabilization of the bath temperature at the equilibrium $T_{r e f}=30^{\circ} \mathrm{C}$, starting from $T_{f}(0)=20^{\circ} \mathrm{C}$ respectively for proportional (in green) and prediction-based (in blue) feedback, both with a gain $k=26$.

certain actuator state defined for constant delays ${ }^{5}$. First, to extend them to the time-varying delay case, we introduce the distributed input $u(x, t)=U(t+D(t)(x-1)), x \in[0,1]$, which enables to rewrite plant (8a) as

$$
\left\{\begin{aligned}
\dot{X}(t) & =A X(t)+B u(0, t) \\
D(t) u_{t}(x, t) & =u_{x}(x, t)+\dot{D}(t)(x-1) u_{x}(x, t) \\
u(1, t) & =U(t)
\end{aligned}\right.
$$

In details, the input delay is now represented as a coupling with a transport partial differential equation (PDE) driven by

${ }^{5}$ This transformation is made to convert the plant $\dot{X}(t)=A X(t)+B u(0, t)$, $D u_{t}(x, t)=u_{x}(x, t)$ with the boundary condition $u(1, t)=U(t)$ into the target system $\dot{X}(t)=(A+B K) X(t)+B w(0, t), D w_{t}(x, t)=w_{x}(x, t)$ with the boundary condition $w(1, t)=0$. 


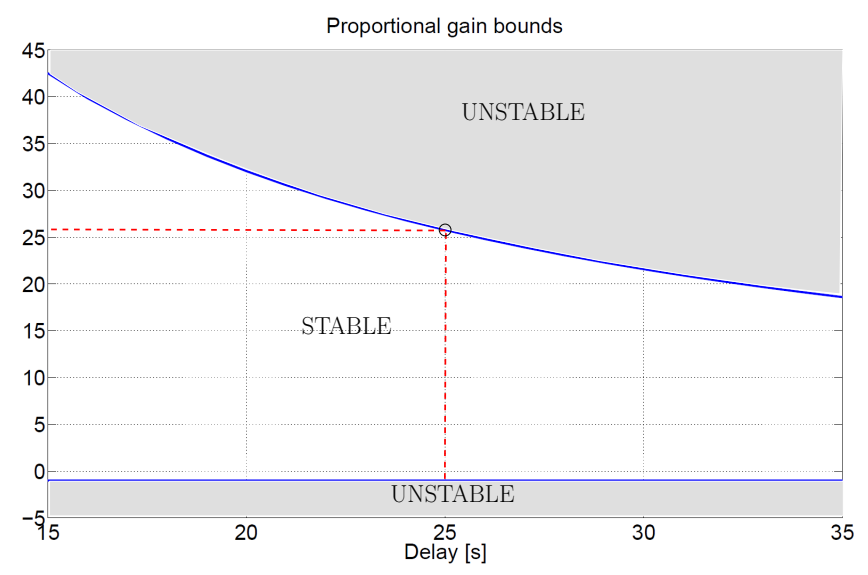

Fig. 4. Stabilizing proportional gains $k$ corresponding to (6) for a delay varying between $15 \mathrm{~s}$ and $35 \mathrm{~s}$. The maximum gain obtained for a $25 \mathrm{~s}$ delay is circled in red.

the input and where the convection speed varies both with space and time.

Pursuing the mentioned approach, we now define the following transformed distributed input, based on the tracking error $\tilde{X}(t)=X(t)-X^{r}$ and the distributed input error $e(x, t)=u(x, t)-U^{r}$,

$$
\begin{aligned}
w(x, t)= & e(x, t)-D(t) K \int_{0}^{x} e^{A D(t)(x-y)} B e(y, t) d y \\
& -K e^{A D(t) x} \tilde{X}(t)
\end{aligned}
$$

This Volterra integral equation of the second kind is designed to fulfill $w(1, t)=0$, compliantly with the control choice formulated earlier. The error plant corresponding to (8a)-(8b) can then be expressed as

$$
\left\{\begin{aligned}
\dot{\tilde{X}}(t) & =(A+B K) \tilde{X}(t)+B w(0, t) \\
D(t) w_{t}(x, t) & =w_{x}(x, t)-\dot{D}(t) D(t) f(x, t) \\
w(1, t) & =0
\end{aligned}\right.
$$

where the function $f$ is defined as

$$
\begin{aligned}
f(x, t)= & \frac{(1-x)}{D(t)} e_{x}(x, t)+K A x e^{A D(t) x} \tilde{X}(t) \\
& +K \int_{0}^{x} e^{A D(t)(x-y)}(I+A D(t)(x-y)) B e(y, t) d y \\
& +K \int_{0}^{x} e^{A D(t)(x-y)} B(y-1) e_{x}(y, t) d y
\end{aligned}
$$

For the Lyapunov analysis below, we also need the governing equation of the spatial derivative of the transformed distributed input $w_{x}$

$$
\begin{aligned}
D(t) w_{x t}(x, t) & =w_{x x}(x, t)-\dot{D}(t) D(t) f_{x}(x, t) \\
w_{x}(1, t) & =\dot{D}(t) D(t) f(1, t)
\end{aligned}
$$

We can now start the Lyapunov analysis and introduce the following Lyapunov-Krasovski functional

$$
\begin{aligned}
V(t)= & \tilde{X}(t)^{T} P \tilde{X}(t)+b_{1} D(t) \int_{0}^{1}(1+x) w(x, t)^{2} d x \\
& +b_{2} D(t) \int_{0}^{1}(1+x) w_{x}(x, t)^{2} d x
\end{aligned}
$$

where the symmetric matrix $P$ satisfies the following Lyapunov equation $P(A+B K)+(A+B K)^{T} P=-Q$ for a given symmetric definite positive matrix $Q$ (we denote $\lambda_{\min }(Q)$ its minimum eigenvalue). Taking a time-derivative of $V$, we get after some integrations by parts

$$
\begin{aligned}
& \dot{V}(t)=-\tilde{X}(t)^{T} Q \tilde{X}(t)+2 \tilde{X}(t)^{T} P B w(0, t)+b_{1}\left(-w(0, t)^{2}\right. \\
& \left.-\|w(t)\|^{2}\right)+b_{2}\left(2 w_{x}(1, t)^{2}-w_{x}(0, t)^{2}-\left\|\hat{w}_{x}(t)\right\|^{2}\right) \\
& -2 b_{1} \dot{D}(t) \int_{0}^{1}(1+x) w(x, t) f(x, t) d x \\
& -2 b_{2} \dot{D}(t) \int_{0}^{1}(1+x) w_{x}(x, t) f_{x}(x, t) d x \\
& +\dot{D}(t)\left(b_{1} \int_{0}^{1}(1+x) w(x, t)^{2} d x+b_{2} \int_{0}^{1}(1+x) w_{x}(x, t)^{2} d x\right) \\
& \leq-\lambda_{\min }(Q)|\tilde{X}(t)|^{2}+2\left|\tilde{X}(t)^{T} P B w(0, t)\right|^{2} \\
& +b_{1}\left(-w(0, t)^{2}-\|w(t)\|^{2}\right. \\
& \left.+2|\dot{D}(t)|\left|\int_{0}^{1}(1+x) w(x, t) f(x, t) d x\right|\right) \\
& +b_{2}\left(2 w_{x}(1, t)^{2}-w_{x}(0, t)^{2}-\left\|w_{x}(t)\right\|^{2}\right. \\
& \left.+2|\dot{D}(t)|\left|\int_{0}^{1}(1+x) w_{x}(x, t) f_{x}(x, t) d x\right|\right) \\
& +|\dot{D}(t)|\left(b_{1} \int_{0}^{1}(1+x) w(x, t)^{2} d x+b_{2} \int_{0}^{1}(1+x) w_{x}(x, t)^{2} d x\right)
\end{aligned}
$$

To bound the remaining positive terms, one can introduce the inverse transformation of (17)

$$
\begin{aligned}
e(x, t)= & w(x, t)+D(t) K \int_{0}^{x} e^{(A+B K) D(t)(x-y)} B w(y, t) d y \\
& +K e^{(A+B K) D(t) x} \tilde{X}(t)
\end{aligned}
$$

and its spatial derivative to obtain the following inequalities, using Young's and Cauchy-Schwartz's inequalities,

$$
\begin{aligned}
& 2\left|\int_{0}^{1}(1+x) w(x, t) f(x, t)\right| d x \\
& \quad \leq M_{1}\left(|\tilde{X}(t)|^{2}+\|w(t)\|^{2}+\left\|w_{x}(t)\right\|^{2}\right) \\
& 2 w_{x}(1, t)^{2} \\
& \quad \leq M_{2}|\dot{D}(t)|^{2}\left(|\tilde{X}(t)|^{2}+\|w(t)\|^{2}+\left\|w_{x}(t)\right\|^{2}\right) \\
& 2\left|\int_{0}^{1}(1+x) w_{x}(x, t) f_{x}(x, t)\right| d x \\
& \leq M_{3}\left(|\tilde{X}(t)|^{2}+\|w(t)\|^{2}+\left\|w_{x}(t)\right\|^{2}+w_{x}(0, t)^{2}\right)
\end{aligned}
$$

where $M_{1}, M_{2}$ and $M_{3}$ are positive constant, the expression of which is not provided here. Using Young's inequality and the previous ones and defining $V_{0}(t)=|\tilde{X}(t)|^{2}+\|w(t)\|^{2}+$ $\left\|w_{x}(t)\right\|^{2}$, it is straightforward to get

$$
\begin{aligned}
& \dot{V}(t) \leq-\frac{\lambda_{\min }(Q)}{2}|\tilde{X}(t)|^{2}-\left(b_{1}-\frac{2|P B|^{2}}{\lambda_{\min }(Q)}\right) w(0, t)^{2} \\
& -b_{1}\|w(t)\|^{2}-b_{2}\left\|w_{x}(t)\right\|^{2}-b_{2}\left(1-M_{3}|\dot{D}(t)|\right) w_{x}(0, t)^{2} \\
& +|\dot{D}(t)|\left(b_{1} M_{1}+b_{2} M_{2}|\dot{D}(t)|+b_{2} M_{3}+2 b_{1}+2 b_{2}\right) V_{0}(t)
\end{aligned}
$$


Consequently, choosing $b_{1} \geq 2|P B|^{2} / \lambda_{\text {min }}(Q)$ and defining

$$
\delta^{*}=\min \left\{\frac{\min \left\{\lambda_{\min }(Q) / 2, b_{1}, b_{2}\right\}}{b_{1} M_{1}+b_{2} M_{2}+b_{2} M_{3}+2 b_{1}+2 b_{2}}, \frac{1}{M_{3}}, 1\right\}
$$

we obtain the existence of a positive constant $\mu$ such that, provided $|\dot{D}(t)|<\delta^{*}, t \geq 0$,

$$
\forall t \in \mathbb{R}_{+}, \quad \dot{V}(t) \leq-\mu V_{0}(t)
$$

Finally, observing that both

$$
\begin{array}{r}
\min \left\{\lambda_{\min }(P), b_{1} \underline{D}, b_{2} \underline{D}\right\} V_{0}(t)=\eta_{1} V_{0}(t) \leq V(t) \\
V(t) \leq \max \left\{\lambda_{\max }(P), 2 b_{1} \bar{D}, 2 b_{2} \bar{D}\right\} V_{0}(t)=\eta_{2} V_{0}(t)
\end{array}
$$

one can deduce that

$$
\forall t \geq 0, \quad V_{0}(t) \leq \frac{\eta_{2}}{\eta_{1}} V_{0}(0) e^{-\frac{\mu}{\eta_{2}} t}
$$

This concludes the proof.

\section{CONCLUSION}

This paper proposes a prediction-based control strategy for a system with an input-dependent delay. This strategy uses a proposed result on control of time-varying delays (Theorem 1) and relates it, to account for the dependence of the delay on the input variable through the considered integral equation modeling the transport phenomenon to a small-gain condition (14).

This control algorithm is based on a novel predictionbased control for linear time-varying input delay. Via a tailored change of time scale, this result has been directly linked to the considered input-dependency of the input delay.

The merits and the implementability of the proposed technique have been highlighted by the presented simulation studies. In particular, comparison with a proportional controller emphasizes its robustness when the delay is increased. This could be reasonably interpreted as a promising way of performance improvements for a large amount of industrial processes involving input-dependent delay.

\section{REFERENCES}

[1] Z. Artstein. Linear systems with delayed controls: a reduction. IEEE Transactions on Automatic Control, 27(4):869-879, 1982.

[2] D. Bresch-Pietri, J. Chauvin, and N. Petit. Adaptive backstepping controller for uncertain systems with unknown input time-delay. application to SI engines. In Proc. of the Conference on Decision and Control, 2010.

[3] D. Bresch-Pietri, J. Chauvin, and N. Petit. Adaptive backstepping for uncertain systems with time-delay on-line update laws. In Proc. of the American Control Conference, 2011.

[4] D. Bresch-Pietri, J. Chauvin, and N. Petit. Output feedback control of time delay systems with adaptation of delay estimate. In Proc. of the IFAC World Congress, 2011.

[5] D. Bresch-Pietri and M. Krstic. Adaptive trajectory tracking despite unknown input delay and plant parameters. Automatica, 45(9):20742081, 2009.

[6] M. Chèbre, Y. Creff, and N. Petit. Feedback control and optimization for the production of commercial fuels by blending. Journal of Process Control, 20(4):441-451, 2010.

[7] K. Gu and S. I. Niculescu. Survey on recent results in the stability and control of time-delay systems. Journal of Dynamic Systems, Measurement, and Control, 125:158, 2003.

[8] Q. L. Han and K. Gu. Stability of linear systems with time-varying delay: a generalized discretized lyapunov functional approach. Asian Journal of Control, 3(3):170-180, 2001.
[9] J. Harmand and D. Dochain. The optimal design of two interconnected (bio) chemical reactors revisited. Computers \& chemical engineering, 30(1):70-82, 2005.

[10] Y. He, Q. G. Wang, C. Lin, and M. Wu. Delay-range-dependent stability for systems with time-varying delay. Automatica, 43(2):371376, 2007.

[11] M. Jankovic. Recursive predictor design for linear systems with time delay. In American Control Conference, 2008, pages 4904-4909. IEEE, 2008.

[12] X. Jiang and Q. L. Han. New stability criteria for linear systems with interval time-varying delay. Automatica, 44(10):2680-2685, 2008.

[13] M. Krstic. Boundary control of PDEs: A course on backstepping designs. Society for Industrial and Applied Mathematics Philadelphia, PA, USA, 2008.

[14] M. Krstic. Lyapunov tools for predictor feedbacks for delay systems: Inverse optimality and robustness to delay mismatch. Automatica, 44(11):2930-2935, 2008.

[15] M. Krstic. Delay compensation for nonlinear, adaptive, and PDE systems. Birkhauser, 2009.

[16] M. Krstic and D. Bresch-Pietri. Delay-adaptive full-state predictor feedback for systems with unknown long actuator delay. In Proceedings of the 2009 conference on American Control Conference, pages 4500-4505, 2009.

[17] Y. Liu and L.-S. Hu. New stabilization method for linear systems with time-varying input delay. In American Control Conference, 2011.

[18] A. Manitius and A. Olbrot. Finite spectrum assignment problem for systems with delays. Automatic Control, IEEE Transactions on, 24(4):541-552, 2002.

[19] S. Mondié and W. Michiels. A safe implementation for finite spectrum assignment: robustness analysis. In Proc. of the 42nd IEEE Conference on Decision and Control (CDC2003), Hawaii, USA, 2003.

[20] Y. S. Moon, P. G. Park, and W. H. Kwon. Robust stabilization of uncertain input-delayed systems using reduction method. Automatica, 37(2):307-312, 2001.

[21] N. Petit, Y. Creff, and P. Rouchon. Motion planning for two classes of nonlinear systems with delays depending on the control. In Proceedings of the 37th IEEE Conference on Decision and Control, volume 1, pages 1007-1011. IEEE, 1998.

[22] J.-P. Richard. Time-delay systems: an overview of some recent advances and open problems. Automatica, 39(10):1667-1694, 2003.

[23] M. Sbarciog, R. De Keyser, S. Cristea, and C. De Prada. Nonlinear Predictive Control of processes with variable time delay. A temperature control case study. In Proc. of the IEEE International Conference on Control Applications, pages 1001-1006. IEEE, 2008.

[24] G. J. Silva, A. Datta, and S. P. Bhattacharyya. PID controllers for time-delay systems. Birkhauser, 2005.

[25] O. J. M. Smith. A controller to overcome dead time. ISA Journal, 6(2):28-33, 1959.

[26] Y. Yildiz, A. Annaswamy, I. Kolmanovsky, and D. Yanakiev. Adaptive Posicast Controller for Time-delay Systems with Relative Degree $\mathrm{n}<=2$. Automatica, 46:279-289, 2010.

[27] D. Yue and Q. L. Han. Delayed feedback control of uncertain systems with time-varying input delay. Automatica, 41(2):233-240, 2005.

[28] Q. C. Zhong. Robust control of time-delay systems. Springer Verlag, 2006. 